\title{
Real-world evidence from a University Hospital system regarding the uptake of adjuvant pertuzumab and/or neratinib before and after their FDA approval
}

\author{
Ericson Stoen ${ }^{1}$ (1) - Jodi Kagihara ${ }^{2} \cdot$ Elena Shagisultanova $^{2} \cdot$ Christine M. Fisher $^{3} \cdot$ Andrew Nicklawsky $^{4} \cdot$ Peter Kabos $^{2}$. \\ Virginia F. Borges ${ }^{2}$. Jennifer R. Diamond ${ }^{2}$
}

Received: 7 October 2020 / Accepted: 5 February 2021 / Published online: 24 February 2021

(c) The Author(s) 2021

\begin{abstract}
Purpose Adjuvant pertuzumab and neratinib are independently FDA-approved for treatment of early-stage HER2-positive breast cancer in combination with or following trastuzumab for one year, respectively. Both agents reduce the risk of recurrence; however, the absolute benefit is modest for many patients with added risk of adverse effects. The purpose of this study was to evaluate the clinical use of adjuvant pertuzumab and neratinib in patients with early-stage HER2-positive breast cancer. Methods Patients diagnosed with stage I-III HER2-positive breast cancer treated with trastuzumab at four University of Colorado Health hospitals between July 2016 and April 2019 were identified. Patient demographics, cancer stage, treatment, and administration of pertuzumab and/or neratinib were obtained.

Results We identified a total of 350 patients who received adjuvant trastuzumab for stage I-III HER2-positive breast cancer; $253(73.1 \%)$ had tumors that were $\geq \mathrm{T} 2$ or node-positive disease. The rate of adjuvant pertuzumab use increased following FDA approval; pertuzumab was administered to the majority of patients with node-positive HER2-positive breast cancer. The use of adjuvant pertuzumab was associated with younger age, premenopausal status, and node-positive disease. Rates of administration of adjuvant neratinib were lower, with only $15.2 \%$ of patients receiving this therapy within 3 months of completing adjuvant trastuzumab.

Conclusion In our cohort of patients treated within a diverse healthcare network, the majority of patients with node-positive HER2-positive breast cancer received adjuvant pertuzumab following FDA approval. The use of adjuvant neratinib was less common, potentially as a result of adverse effects, prolongation of therapy, previous administration of adjuvant pertuzumab, and modest benefit.
\end{abstract}

Keywords Breast cancer $\cdot$ HER2 $\cdot$ Pertuzumab - Neratinib - Adjuvant therapy

\section{Introduction}

Ericson Stoen

ericson.stoen@cuanschutz.edu

1 Department of Internal Medicine, University of Colorado School of Medicine, 12401 East 17th Avenue, Mailstop F-782, Aurora, CO 80045, USA

2 Division of Medical Oncology, University of Colorado School of Medicine, Aurora, CO, USA

3 Department of Radiation Oncology, University of Colorado School of Medicine, Aurora, CO, USA

4 Department of Pediatrics, University of Colorado School of Medicine, Aurora, CO, USA
Breast cancer is the most common cancer in women and approximately $20 \%$ of breast cancers overexpress human epidermal growth factor receptor 2 (HER2) [1-3]. HER2positive breast cancer was historically associated with a worse prognosis; however, the addition of trastuzumab to adjuvant chemotherapy significantly reduced the risk of disease recurrence and death [4-8]. Both pertuzumab and neratinib have been independently shown to further reduce the risk of recurrence in patients with HER2-positive breast cancer treated with chemotherapy and trastuzumab for one year, though the magnitude of benefit is much smaller than the initial benefit gained from targeting HER2 with the addition of trastuzumab $[9,10]$. 
Pertuzumab is a humanized recombinant anti-HER2 monoclonal antibody that inhibits dimerization of HER2 with other HER family receptors and is synergistic with trastuzumab [11-13]. The addition of pertuzumab to docetaxel and trastuzumab in the first-line, HER2-positive metastatic setting led to an improvement in progression-free survival (PFS) and overall survival (OS) and remains the standard of care for these patients [12]. In the neoadjuvant setting, the addition of pertuzumab to trastuzumab-containing chemotherapy led to improvements in pathologic complete response (pCR) and disease-free survival (DFS) and remains the standard of care for neoadjuvant therapy in high-risk patients $[13,14]$. In patients with node-positive or highrisk node-negative operable HER2-positive breast cancer, the APHINITY trial found that the addition of pertuzumab improved the 3-year rate of invasive disease-free survival (iDFS) from $93.2 \%$ in the placebo group to $94.1 \%$ in the pertuzumab group (HR 0.81; 95\% CI 0.66-1.00, $P=0.045$ ). The benefit was greater in patients with node-positive disease, with a 3-year iDFS rate of $90.2 \%$ in the placebo group and $92.0 \%$ in those receiving pertuzumab (HR 0.77; 95\% CI $0.62-0.96, P=0.02)$. Cardiac events were low in both groups and diarrhea was more common with pertuzumab [9]. With 6-year follow-up, the addition of pertuzumab did not result in a statistically significant difference in OS, but continued follow-up is planned [15].

Neratinib is an oral small-molecule tyrosine kinase inhibitor of HER1, HER2, and HER4 with single-agent and combination activity in patients with metastatic HER2-positive breast cancer previously treated with trastuzumab [10, 16-18]. In the neoadjuvant setting, the addition of neratinib to standard chemotherapy with trastuzumab resulted in an improvement in the pCR rate in HER2-positive, hormone receptor-negative patients in the I-SPY2 trial [18]. Based on these results, neratinib was evaluated in the adjuvant setting in the phase III, randomized, placebo-controlled ExteNET study, which assessed the benefit of addition of 1 year of adjuvant neratinib after standard chemotherapy and trastuzumab in patients with early-stage, high-risk HER2-positive breast cancer. The addition of neratinib resulted in an improvement in the 5-year iDFS rate versus placebo $(90.2 \%$ vs. $87.7 \%$, HR 0.73 ; $95 \%$ CI $0.57-0.92, P=0.0083)$. The magnitude of benefit in 5-year iDFS was greater in the subgroup of patients with hormone receptor-positive disease (HR 0.60; 95\% CI 0.43-0.83) compared to a nonsignificant difference in patients with hormone receptor-negative disease (HR 0.95; 95\% CI 0.66-1.35) [10]. In the ExteNET trial, patients treated with adjuvant neratinib had a significantly higher rate of grade 3 diarrhea compared to placebo (40\% versus $2 \%$ ). No patients enrolled in ExteNET received prior pertuzumab and no patients enrolled in APHINITY received adjuvant neratinib $[9,10]$.
Given the simultaneous approval of both pertuzumab and neratinib for the adjuvant treatment of high-risk, earlystage, HER2-positive breast cancer, the added toxicity with these agents, and the modest improvement in recurrence rates overall, the decision to incorporate these agents into standard practice is complex. The purpose of this study was to evaluate the use of adjuvant pertuzumab and neratinib in patients with high-risk, early-stage HER2-positive breast cancer within the University of Colorado Health system.

\section{Methods}

Female patients aged 18-84 years old diagnosed with stage I-III HER2-positive breast cancer treated with trastuzumab at multiple centers within the University of Colorado Health system between July 2016 and April 2019 were identified using an electronic data capture system. This study was performed following local institutional review board approval and all data were stored in a secure online database. HER2positivity was defined by local pathology report using ASCO/CAP guidelines [19]. Patients pregnant at the time of treatment were excluded. Patients must have had at least two visits with a provider within the system to ensure adequate follow-up. Patients received treatment at four centers in the University of Colorado Health system. Chart review was performed for all patients using the electronic medical record. Baseline characteristics including age; date of diagnosis; stage at diagnosis (highest clinical or pathologic stage); estrogen receptor expression (as assessed by local pathology report); menopausal status (by physician documentation); cancer treatment including surgery, radiation, chemotherapy; and receipt of adjuvant pertuzumab and/or neratinib were collected. Patients receiving at least one dose of pertuzumab following the completion of neoadjuvant or adjuvant chemotherapy were included as having received adjuvant pertuzumab. The duration of adjuvant pertuzumab was determined and receipt of 12 months was considered a complete course (including neoadjuvant or adjuvant pertuzumab administered in combination with chemotherapy). Patients receiving any duration of neratinib within 3 months of completing adjuvant trastuzumab were considered as having received adjuvant neratinib. Given the high incidence of neratinib-induced diarrhea, receipt of concomitant antidiarrheal prophylaxis was also collected.

Patient characteristics were analyzed using percentages for categorical variables and mean for continuous variables. Associations between patient characteristics and administration of pertuzumab or neratinib were performed using the 2-sided Chi-squared test for categorical variables. For variables with small sample size, data were consolidated and reported as pooled. An alpha value of 0.05 was used 
for all statistical analysis, which was considered statistically significant.

\section{Results}

\section{Baseline characteristics}

In our cohort, 350 patients were identified with stage I-III HER2-positive breast cancer who received trastuzumab and were included in our analysis. Of those, 346 had adequate follow-up to be included in our dataset. In order to focus our analysis on patients with high-risk, HER2positive breast cancer, our analysis was limited to the 253 patients $(73.1 \%)$ with tumor stage $\geq \mathrm{T} 2$ or node-positive disease at diagnosis. Patients with disease that was T1 or node-negative were not included in our analysis.

Tables 1 and 2 depict baseline patient characteristics. The mean age of patients with high-risk, earlystage HER2-positive breast cancer was 53.2 years (range 24-82), and 171 of 253 (67.6\%) had node-positive disease. The majority of patients had hormone receptor-positive disease $(172 / 253,68.0 \%)$, and 133 of $253(52.6 \%)$ were postmenopausal. Neoadjuvant chemotherapy was administered to $73.9 \%$ of patients in this cohort (187/253 patients).

\section{Patients receiving adjuvant pertuzumab}

High-risk, early-stage HER2-positive breast cancer patients receiving adjuvant pertuzumab were younger than patients who did not receive adjuvant pertuzumab (mean age of 48.4 compared to 55.8 years, $P<0.0001$ ) (Table 1). There was also a higher incidence of utilization of adjuvant pertuzumab in premenopausal patients $(P=0.0053)$. Hormone receptor positivity was not associated with administration of adjuvant pertuzumab (70.7\% [65/92] versus $66.5 \%$ [107/161], $P=0.4917$ ), nor was the likelihood of pCR after neoadjuvant chemotherapy, if given (42.5\% [31/73] versus $44.7 \%$ [51/114], $P=0.7601)$. Among patients that received adjuvant pertuzumab, $76.1 \%$ (70/92) had node-positive disease. Of all high-risk patients studied, 36.4\% (92/253) received adjuvant pertuzumab, including $39.8 \%$ (68/171) of patients with node-positive disease and $26.8 \%$ (22/82) of patients with node-negative, $\geq \mathrm{T} 2$ disease (Table 2 ).

The rate of adjuvant pertuzumab administration increased over time from July 2016 to December 2018 following the publication of the APHINITY trial results in July 2017 and the FDA approval of adjuvant pertuzumab in high-risk earlystage breast cancer in December 2017 (Fig. 1). At the peak of administration in our cohort, approximately two-thirds of patients with node-positive, HER2-positive disease received adjuvant pertuzumab. The average duration of therapy was
10.7 months and $83.7 \%$ of patients received at least 8 months of therapy.

\section{Patients receiving adjuvant neratinib}

The mean age of patients with high-risk, early-stage HER2positive breast cancer who received adjuvant neratinib was 49.1 compared to 53.8 years for those who did not $(P=0.0679)$ (Table 3). Hormone receptor status did not correlate with neratinib use; however, there was a trend toward increased use in hormone receptor-positive patients. Patients receiving adjuvant neratinib were less likely to have achieved $\mathrm{pCR}$ following neoadjuvant chemotherapy (24.0\% [6/25] versus $47.2 \%$ [77/163], $P=0.0297$ ).

In our dataset of patients with $\geq \mathrm{T} 2$ or node-positive disease, only 30 of 253 patients $(11.9 \%)$ received adjuvant neratinib. Among patients with node-positive disease, $15.2 \%$ (26/171) received adjuvant neratinib; among patients with $\geq \mathrm{T} 2$, node-negative disease, $4.9 \%$ (4/82) received adjuvant neratinib (Table 4). The majority of patients (86.7\%) receiving adjuvant neratinib had node-positive disease. Rates of administration of adjuvant neratinib increased steadily following the publication of the ExteNET trial results in November 2017, reaching a peak in May 2018 when approximately $30 \%$ of patients with high-risk, early-stage HER2positive breast cancer were treated with adjuvant neratinib (Fig. 2). No patients received adjuvant neratinib prior to FDA approval. For patients receiving adjuvant neratinib, the average time from completion of adjuvant trastuzumab to initiation of adjuvant neratinib was 2.97 months. All patients receiving adjuvant neratinib were also prescribed diarrheal prophylaxis with loperamide. Additional anti-diarrheal agents included colestipol (66.7\%), atropine/diphenoxylate (Lomotil) (3\%), cholestyramine (6\%), and budesonide (3\%).

We identified 14 patients (5.5\%) of the 253 identified with high-risk, early-stage HER2-positive breast disease who received both adjuvant pertuzumab and adjuvant neratinib. These patients had many high-risk features including younger age (average 42.9 years); T2 or higher tumors (14/14), and node-positivity (12/14, 85.7\%). The majority of patients receiving both pertuzumab and neratinib were HR-positive $(12 / 14,85.7 \%)$ and of the 11 that received neoadjuvant chemotherapy, 7 (63.6\%) did not achieve a pCR.

\section{Discussion}

In our study, we evaluated the practice patterns for the use of adjuvant pertuzumab and neratinib in patients with high-risk, early-stage HER2-positive breast cancer as defined as $\geq \mathrm{T} 2$ or node-positive disease. We found that the majority of patients with node-positive disease went on to receive adjuvant pertuzumab following FDA approval in the adjuvant 
Table 1 Baseline characteristics of patients with high-risk early-stage HER2-positive breast cancer receiving or not receiving adjuvant pertuzumab

\begin{tabular}{|c|c|c|c|c|}
\hline & $\begin{array}{l}\text { All patients } \\
(N=253)\end{array}$ & $\begin{array}{l}\text { Received pertu- } \\
\text { zumab }(N=92)\end{array}$ & $\begin{array}{l}\text { Did not receive pertu- } \\
\text { zumab }(N=161)\end{array}$ & $P$-value* \\
\hline Age (years) & & & & $<0.0001$ \\
\hline Mean & 53.2 & 48.4 & 55.8 & \\
\hline Range & $24-82$ & $31-73$ & $24-82$ & \\
\hline Standard deviation & 13.31 & 10.99 & 13.78 & \\
\hline Practice Setting & & & & 0.0442 \\
\hline Academic & $94(37.2 \%)$ & $42(45.7 \%)$ & $53(32.9 \%)$ & \\
\hline Community & $159(62.8 \%)$ & $50(54.3 \%)$ & $108(67.1 \%)$ & \\
\hline AJCC Tumor Size & & & & 0.1857 \\
\hline $\mathrm{T} 1$ & $43(17.0 \%)$ & $13(14.1 \%)$ & $31(19.2 \%)$ & \\
\hline $\mathrm{T} 2$ & $158(62.5 \%)$ & $53(57.6 \%)$ & $105(65.2 \%)$ & \\
\hline T3 & $34(13.4 \%)$ & $17(18.5 \%)$ & $17(10.6 \%)$ & \\
\hline $\mathrm{T} 4$ & $16(6.3 \%)$ & $8(8.7 \%)$ & $7(4.3 \%)$ & \\
\hline $\mathrm{TX}$ & $2(0.8 \%)$ & $1(1.1 \%)$ & $1(0.6 \%)$ & \\
\hline Nodal Status & & & & 0.0356 \\
\hline No & $82(32.4 \%)$ & $22(23.9 \%)$ & $62(38.5 \%)$ & \\
\hline N1 & $139(54.9 \%)$ & $52(56.5 \%)$ & $85(52.8 \%)$ & \\
\hline $\mathrm{N} 2$ & $15(5.9 \%)$ & $9(9.8 \%)$ & $6(3.7 \%)$ & \\
\hline N3 & $17(6.7 \%)$ & $9(9.8 \%)$ & $8(5.0 \%)$ & \\
\hline Hormone Status & & & & 0.4917 \\
\hline ER and/or PR-positive & $172(68.0 \%)$ & $65(70.7 \%)$ & $107(66.5 \%)$ & \\
\hline ER/PR-negative & $81(32.0 \%)$ & $27(29.3 \%)$ & $54(33.5 \%)$ & \\
\hline Menopausal Status & & & & 0.0053 \\
\hline Premenopausal & $97(38.3 \%)$ & $47(51.1 \%)$ & $51(31.7 \%)$ & \\
\hline Postmenopausal & $133(52.6 \%)$ & $36(39.1 \%)$ & $96(59.6 \%)$ & \\
\hline Unknown & $23(9.1 \%)$ & $9(9.8 \%)$ & $14(8.7 \%)$ & \\
\hline Received Radiation & & & & 0.1134 \\
\hline Yes & $193(76.3 \%)$ & $75(81.5 \%)$ & $117(72.7 \%)$ & \\
\hline No & $60(23.7 \%)$ & $17(18.5 \%)$ & $44(27.3 \%)$ & \\
\hline \multicolumn{5}{|c|}{ Received Neoadjuvant Chemotherapy } \\
\hline Yes & $187(73.9 \%)$ & $73(79.3 \%)$ & $114(70.8 \%)$ & \\
\hline No & $66(26.1 \%)$ & $19(20.7 \%)$ & $47(29.2 \%)$ & \\
\hline \multicolumn{4}{|c|}{ pCR After Neoadjuvant Chemotherapy } & 0.7601 \\
\hline Yes & $83(44.4 \%)$ & $31(42.5 \%)$ & $51(44.7 \%)$ & \\
\hline No & $104(55.6 \%)$ & $42(57.5 \%)$ & $63(55.3 \%)$ & \\
\hline
\end{tabular}

* $P$-value calculated using Chi-squared analysis comparing pertuzumab to no pertuzumab groups setting, and patients with node-negative, $\geq \mathrm{T} 2$ disease were less likely to receive this treatment. Patients receiving adjuvant pertuzumab were younger, and more likely to be premenopausal and node-positive. Adjuvant neratinib was less commonly used in this cohort and was administered to $11.9 \%$ of patients overall and $15.2 \%$ of patients with node-positive disease. There was a trend toward higher use in patients with HR-positive disease; however, the number of patients receiving adjuvant neratinib was small in our dataset, which limited this analysis.

We observed that patients with node-positive disease were most likely to receive adjuvant pertuzumab, consistent 
Table 2 Use of adjuvant pertuzumab by cancer stage

\begin{tabular}{llll}
\hline & $\begin{array}{l}\text { Node-positive and/or } \\
\text { tumor } \geq \mathrm{T} 2(N=253)\end{array}$ & Node-positive $(N=171)$ & $\begin{array}{l}\text { Node-negative, } \\
\text { tumor } \geq \mathrm{T} 2 \\
(N=82)\end{array}$ \\
\hline $\begin{array}{l}\text { Adjuvant pertuzumab } \\
\text { No adjuvant pertuzumab }\end{array}$ & $92(36.4 \%)$ & $70(40.9 \%)$ & $22(26.8 \%)$ \\
\hline
\end{tabular}

with the benefit observed in this patient population in the APHINITY trial [9]. The observed practice patterns are consistent with both NCCN and ASCO guidelines recommending the consideration of adjuvant pertuzumab in patients with node-positive, HER2-positive breast cancer [20, 21].

Interestingly, we observed a relatively high rate of administration of adjuvant pertuzumab in high-risk patients prior to its FDA approval in the adjuvant setting. One reason for this may be the magnitude of benefit observed with the addition of pertuzumab to treatment regimens in the metastatic and neoadjuvant settings which supported the evaluation of pertuzumab in the APHINITY trial. As pertuzumab was commercially available at the time and used in the neoadjuvant setting for many of these patients, providers likely had the opportunity to continue pertuzumab in the adjuvant setting for patients identified as high risk of recurrence while awaiting the results of APHINITY. Following publication of the APHINITY trial results, rates of administration of adjuvant pertuzumab further increased in node-positive patients. In the final quarter of our analysis, a decline in the rate of use of adjuvant pertuzumab was observed which correlated with publication of the KATHERINE trial and a change in our standard of care to administer ado-trastuzumab emtansine in the adjuvant setting for patients who received neoadjuvant chemotherapy without pCR [22].

The mean duration of adjuvant pertuzumab for patients receiving it in our study was 10.7 months and the vast majority of patients received at least 8 months of therapy. Treatment discontinuation prior to the planned 12 months of therapy most commonly occurred due to treatment-related toxicities. The most frequently reported high-grade adverse events in clinical trials of pertuzumab in combination with chemotherapy and trastuzumab were diarrhea, anemia, and neutropenia. In the APHINITY trial, the rate of grade 3 or higher diarrhea was $9.8 \%$ in patients receiving pertuzumab compared to $3.7 \%$ in the placebo group. However, rates of high-grade diarrhea of up to $37 \%$ were reported in a retrospective study of patients receiving pertuzumab in the neoadjuvant or metastatic settings [23]. Congestive heart failure attributable to pertuzumab is rare [24]; however, it

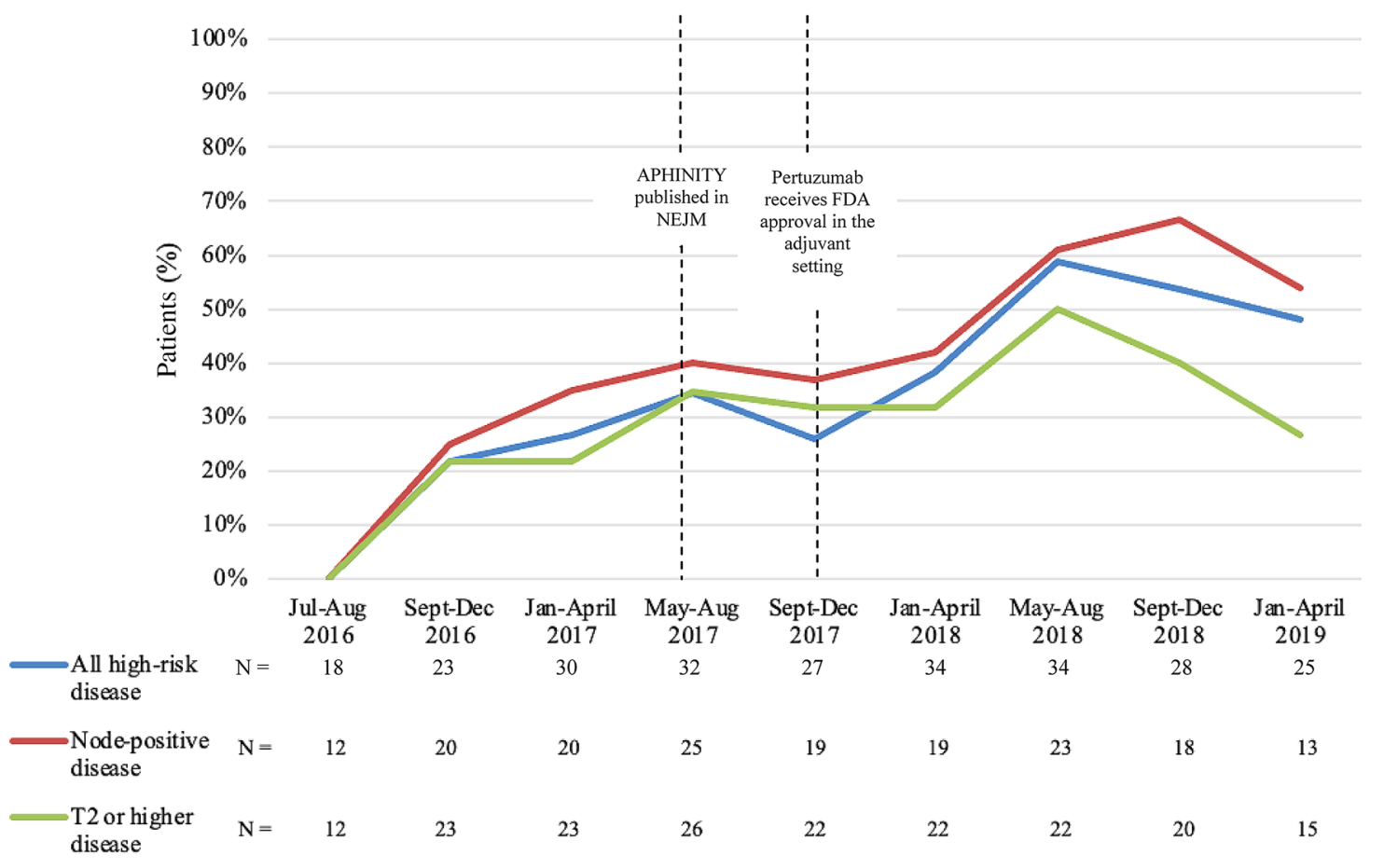

Fig. 1 Rates of initiation of adjuvant pertuzumab in patients with high-risk, early-stage HER2-positive breast cancer over time 
Table 3 Baseline characteristics of patients with high-risk early-stage HER2-positive breast cancer receiving or not receiving adjuvant neratinib

\begin{tabular}{|c|c|c|c|c|}
\hline & All patients $(N=253)$ & $\begin{array}{l}\text { Received ner- } \\
\text { atinib }(N=30)\end{array}$ & $\begin{array}{l}\text { Did not receive ner- } \\
\text { atinib }(N=223)\end{array}$ & $P$-value* \\
\hline Age (years) & & & & 0.0679 \\
\hline Mean & 53.2 & 49.1 & 53.8 & \\
\hline Range & $24-82$ & $29-77$ & $24-82$ & \\
\hline Standard deviation & 13.31 & 13.13 & 13.26 & \\
\hline Location of Care & & & & 0.0871 \\
\hline Academic & $94(37.2 \%)$ & $17(56.7 \%)$ & $77(34.5 \%)$ & \\
\hline Community & $159(62.8 \%)$ & $13(43.3 \%)$ & $146(65.5 \%)$ & \\
\hline AJCC Tumor Size & & & & 0.0027 \\
\hline $\mathrm{T} 1$ & $43(17.0 \%)$ & $4(13.3 \%)$ & $39(17.5 \%)$ & \\
\hline $\mathrm{T} 2$ & $158(62.5 \%)$ & $13(43.3 \%)$ & $145(65.0 \%)$ & \\
\hline $\mathrm{T} 3$ & $34(13.4 \%)$ & $11(36.7 \%)$ & $23(10.3 \%)$ & \\
\hline $\mathrm{T} 4$ & $16(6.3 \%)$ & $2(6.7 \%)$ & $14(6.3 \%)$ & \\
\hline $\mathrm{TX}$ & $2(0.8 \%)$ & 0 & $2(0.9 \%)$ & \\
\hline Nodal Status & & & & 0.0180 \\
\hline No & $82(32.4 \%)$ & $4(13.3 \%)$ & $78(35.0 \%)$ & \\
\hline N1 & $139(54.9 \%)$ & $20(66.7 \%)$ & $119(53.4 \%)$ & \\
\hline $\mathrm{N} 2$ & $15(5.9 \%)$ & $1(3.3 \%)$ & $14(6.3 \%)$ & \\
\hline N3 & $17(6.7 \%)$ & $5(16.7 \%)$ & $12(5.4 \%)$ & \\
\hline Hormone Status & & & & 0.2776 \\
\hline ER and/or PR-positive & $172(68.0 \%)$ & $23(76.7 \%)$ & $149(66.8 \%)$ & \\
\hline ER/PR-negative & $81(32.0 \%)$ & $7(23.3 \%)$ & $74(33.2 \%)$ & \\
\hline Menopausal Status & & & & 0.1504 \\
\hline Premenopausal & $97(38.3 \%)$ & $16(53.3 \%)$ & $81(36.3 \%)$ & \\
\hline Postmenopausal & $133(52.6 \%)$ & $13(43.3 \%)$ & $120(53.8 \%)$ & \\
\hline Unknown & $23(9.1 \%)$ & $1(3.3 \%)$ & $22(9.9 \%)$ & \\
\hline Received Radiation & & & & 0.0599 \\
\hline Yes & $193(76.3 \%)$ & $27(90.0 \%)$ & $166(74.4 \%)$ & \\
\hline No & $60(23.7 \%)$ & $3(10.0 \%)$ & $57(25.6 \%)$ & \\
\hline \multicolumn{5}{|c|}{ Received Neoadjuvant Chemotherapy } \\
\hline Yes & $187(73.9 \%)$ & $25(83.3 \%)$ & $162(72.6 \%)$ & \\
\hline No & $66(26.1 \%)$ & $5(16.7 \%)$ & $61(27.4 \%)$ & \\
\hline \multicolumn{4}{|c|}{ pCR After Neoadjuvant Chemotherapy } & 0.0297 \\
\hline Yes & $83(44.4 \%)$ & $6(24.0 \%)$ & $77(47.5 \%)$ & \\
\hline No & $104(55.6 \%)$ & $19(76.0 \%)$ & $85(52.5 \%)$ & \\
\hline
\end{tabular}

* $P$-value calculated using Chi-squared analysis comparing neratinib to no neratinib groups was more common in those receiving pertuzumab $(0.6 \%)$ versus placebo (0.2\%) in APHINITY [9].

While the improvement in iDFS with adjuvant pertuzumab demonstrated in the APHINITY trial may be considered modest, its use was predicted to be cost-effective based on quality-adjusted life years gained by preventing disease recurrence [25]. However, the addition of potential treatment-related toxicity is likely to impact treatment decisions as clinicians weigh the individualized benefit in reduction of risk of recurrence with toxicity. Further work to
Table 4 Use of adjuvant neratinib by cancer stage

\begin{tabular}{llll}
\hline & $\begin{array}{l}\text { Node-positive and/or } \\
\text { tumor } \geq \mathrm{T} 2(N=253)\end{array}$ & Node-positive $(N=171)$ & $\begin{array}{l}\text { Node-negative, } \\
\text { tumor } \geq \mathrm{T} 2 \\
(N=82)\end{array}$ \\
\hline Adjuvant neratinib & $30(11.9 \%)$ & $26(15.2 \%)$ & $4(4.9 \%)$ \\
No adjuvant neratinib & $223(88.1 \%)$ & $145(84.8 \%)$ & $78(95.1 \%)$ \\
\hline
\end{tabular}


identify factors beyond node-positive disease that may predict a high risk of metastatic recurrence following chemotherapy and trastuzumab and increased benefit from adjuvant pertuzumab are likely planned as the APHINITY trial data continue to mature and will be helpful in making these decisions in the future.

In contrast to pertuzumab, the majority of patients in our dataset did not go on to receive adjuvant neratinib, although its use was more common in node-positive patients. Our analysis may be limited by short-term follow-up of patients for at least 3 months following completion of adjuvant trastuzumab to determine if adjuvant neratinib would be used. The ExteNET trial allowed patients to enroll within 12 months of completing adjuvant trastuzumab; however, the benefit was greater in patients that initiated therapy closer to completion of adjuvant trastuzumab, particularly within 6 months [10, 26, 27].

In the ExteNET trial, diarrhea was the most common neratinib-related adverse event with $>40 \%$ of patients experiencing grade 3 or 4 diarrhea. Patients were offered loperamide as needed for symptomatic management, though prophylaxis was not initially administered per protocol [10]. Neratinib-related diarrhea typically occurs within the first cycle of therapy and often leads to dose reductions in the absence of anti-diarrheal prophylaxis [28]. We now know based on the results of the CONTROL trial that scheduled loperamide plus colestipol is effective anti-diarrheal prophylaxis in patients receiving neratinib [29]. In our study, all patients receiving neratinib received loperamide, and about two-thirds also received prophylactic colestipol. This is likely a result of provider education and standardized therapy care plans that include anti-diarrheal prophylaxis that are used across our practice sites. In further contrast to the utilization of adjuvant pertuzumab, Schwartz et al. found that adjuvant neratinib was not cost-effective, even in this high-risk group, based on the 5-year data from the ExteNET study [30]. Patients and providers may have concerns about potential toxicity with neratinib and a modest decrease in the risk of recurrence and ultimately choose not to initiate therapy.

Very few patients received both adjuvant pertuzumab and neratinib in our study which is consistent with a lack of data for the benefit of adjuvant neratinib in patients receiving adjuvant pertuzumab and vice versa.

Our study has several limitations including a relatively small sample size and treatment within one hospital system with standardized cancer therapy administration order sets. Patients were followed for only 3 months after completion of adjuvant trastuzumab; therefore, the rate of adjuvant neratinib administration may be underestimated. Ongoing follow-up of this cohort of patients is planned to further understand the shifting practice patterns of adjuvant therapy in HER2-positive breast cancer with the availability of new agents and potentially validation of patient selection strategies to maximize benefit and minimize toxicity for patients less likely to experience recurrence.

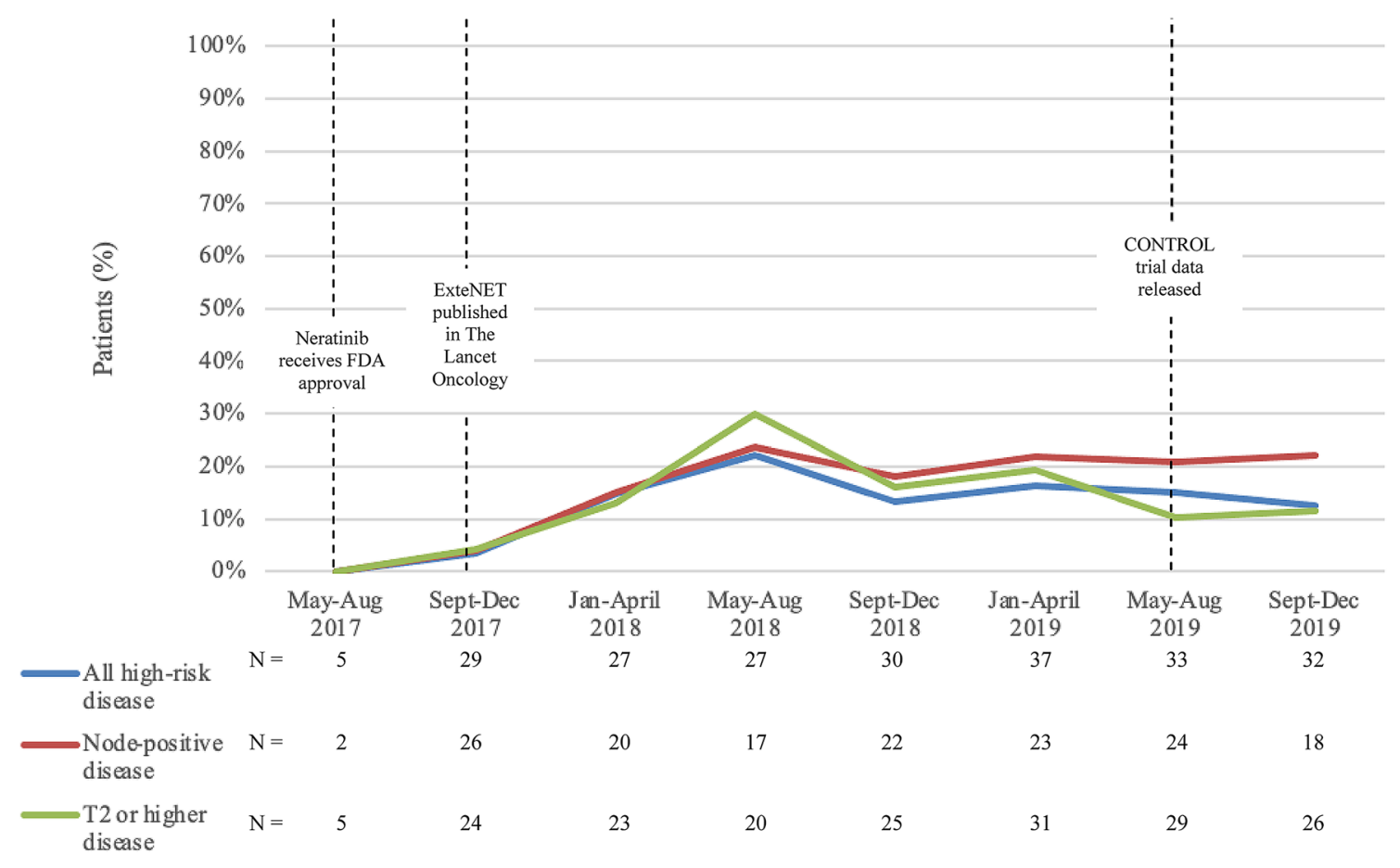

Fig. 2 Rates of initiation of adjuvant neratinib in patients with high-risk, early-stage HER2-positive breast cancer over time 
Author contributions ES and JRD conceived and designed the presented idea and, with assistance from JK, developed the methodology. ES obtained and analyzed the data and AN verified the analytical methods. ES and JRD wrote the manuscript in consultation with JK, ES, CF, $\mathrm{PK}$, and VFB. All authors provided critical feedback and helped shape the research, analysis, and manuscript.

\section{Funding Not applicable.}

Data availability The datasets generated during and/or analyzed during the current study are available from the corresponding author on reasonable request.

Code availability Not applicable.

\section{Compliance with ethical standards}

Conflict of interest Dr. Peter Kabos has received funding from Pfizer, Lilly, Astra Zeneca, Radius Health, Sanofi, and Genentech. The remaining authors declare that they have no conflict of interest.

Ethical approval All procedures were in accordance with the ethical standards of the institutional research committee and with the 1964 Helsinki declaration and its later amendments or comparable ethical standards.

Informed consent Necessity of informed consent was waived as the above investigation was a retrospective chart review.

Open Access This article is licensed under a Creative Commons Attribution 4.0 International License, which permits use, sharing, adaptation, distribution and reproduction in any medium or format, as long as you give appropriate credit to the original author(s) and the source, provide a link to the Creative Commons licence, and indicate if changes were made. The images or other third party material in this article are included in the article's Creative Commons licence, unless indicated otherwise in a credit line to the material. If material is not included in the article's Creative Commons licence and your intended use is not permitted by statutory regulation or exceeds the permitted use, you will need to obtain permission directly from the copyright holder. To view a copy of this licence, visit http://creativecommons.org/licenses/by/4.0/.

\section{References}

1. Slamon D, Clark G, Wong S et al (1987) Human breast cancer: Correlation of relapse and survival with amplification of the HER-2/neu oncogene. Science 235(4785):177-182

2. Moja L, Tagliabue L, Balduzzi S et al (2012) Trastuzumab containing regimens for early breast cancer. Cochrane Database of Syst Rev. https://doi.org/10.1002/14651858.CD006243.pub2

3. Brufsky A (2010) Trastuzumab-based therapy for patients with HER2-positive breast cancer. Am J Clin Oncol 33(2):186-195

4. Gonzalez-Angulo AM, Litton JK, Broglio KR et al (2009) High Risk of Recurrence for Patients With Breast Cancer Who Have Human Epidermal Growth Factor Receptor 2-Positive, Node-Negative Tumors $1 \mathrm{~cm}$ or Smaller. J Clin Oncol 27(34):5700-5706

5. Cameron D, Piccart-Gebhart M, Gelber R et al (2017) 11 years' follow-up of trastuzumab after adjuvant chemotherapy in HER2-positive early breast cancer: Final analysis of the
HERceptin Adjuvant (HERA) trial. Lancet (North American Edition) 389(10075):1195-1205

6. Perez E, Romond E, Suman V et al (2014) Trastuzumab plus adjuvant chemotherapy for human epidermal growth factor receptor 2-positive breast cancer: Planned joint analysis of overall survival from NSABP B-31 and NCCTG N9831. J Clin Oncol 32(33):3744-3752

7. Slamon D, Eiermann W, Robert N et al (2011) Adjuvant trastuzumab in HER2-positive breast cancer. N Eng J Med 365(14):1273-1283

8. Escrivá-De-Romaní S, Arumí M, Bellet M et al (2018) HER2positive breast cancer: Current and new therapeutic strategies. Breast 39:80-88

9. von Minckwitz G, Procter M, de Azambuja E et al (2017) Adjuvant pertuzumab and trastuzumab in early HER2-positive breast cancer. N Eng J Med 377(2):122-131

10. Martin M, Holmes FA, Ejlertsen B et al (2017) Neratinib after trastuzumab-based adjuvant therapy in HER2-positive breast cancer (ExteNET): 5-year analysis of a randomized, double-blind, placebo-controlled, phase 3 trial. Lancet Oncol 18:1688-1700

11. Nami B, Maadi H, Wang Z (2019) The effects of pertuzumab and its combination with trastuzumab on HER2 homodimerization and phosphorylation. Cancers 11(3):375-395

12. Baselga J, Cortés J, Kim SB et al (2012) Pertuzumab plus trastuzumab plus docetaxel for metastatic breast cancer. N Engl J Med 366(2):109-119

13. Gianni L, Pienkowski T, Im YH et al (2012) Efficacy and safety of neoadjuvant pertuzumab and trastuzumab in women with locally advanced, inflammatory, or early HER2-positive breast cancer (NeoSphere): a randomised multicentre, open-label, phase 2 trial. Lancet Oncol 13(1):25-32

14. Gianni L, Pienkowski T, Im Y et al (2016) 5-year analysis of neoadjuvant pertuzumab and trastuzumab in patients with locally advanced, inflammatory, or early-stage HER2-positive breast cancer (NeoSphere): A multicentre, open-label, phase 2 randomised trial. Lancet Oncol 17(6):791-800

15. Piccart M, Procter M, Fumagalli D et al (2019) Interim overall survival analysis of APHINITY (BIG 4-11): A randomized multicenter, double-blind, placebo-controlled trial comparing chemotherapy plus trastuzumab plus pertuzumab versus chemotherapy plus trastuzumab plus placebo as adjuvant therapy in patients with operable HER2-positive early breast cancer [Abstract]. San Antonio Breast Cancer Symposium. https://doi. org/10.1158/1538-7445.SABCS19-GS1-04

16. Burstein HJ, Sun Y, Dirix LY et al (2010) Neratinib, an irreversible ErbB receptor tyrosine kinase inhibitor, in patients with advanced ErbB2-positive breast cancer. J Clin Oncol 28(8):1301-1307

17. Debiasi M, Polanczyk CA, Ziegelmann P et al (2018) Efficacy of anti-HER2 agents in combination with adjuvant or neoadjuvant chemotherapy for early and locally advanced HER2-positive breast cancer patients: A network meta-analysis. Frontiers in Oncology 8(156):1-8

18. Park JW, Liu MC, Yee D et al (2016) Adaptive randomization of neratinib in early breast cancer. N Engl J Med 375(1):11-22

19. Wolff AC, Hammond EH, Allison KH et al (2018) Human Epidermal Growth Factor Receptor 2 Testing in Breast Cancer. Arch Pathol Lab Med 142:1364-1382

20. Gradishar, W.J., Anderson, B.O., Abraham, J., et al. NCCN Clinical Practice Guidelines in Oncology: Breast Cancer. Version 4.2020. Available at: NCCN.org.

21. Denduluri N, Chavez-Macgregor M, Telli ML et al (2018) Selection of optimal adjuvant chemotherapy and targeted therapy for early breast cancer: ASCO clinical practice guideline focused update. J Clin Oncol 36(23):2433-2443 
22. von Minckwitz G, Huang C, Mano MS et al (2019) Trastuzumab Emtansine for Residual Invasive HER2-Positive Breast Cancer. N Engl J Med 380(7):617-628

23. Yankulina O, Zullo AR, Cabral SE et al (2019) Pertuzumabassociated diarrhea in HER2/neu-positive breast cancer patients: A comparison of trials to actual practice. Journal of Oncology Pharmacy Practice 26(4):912-917

24. Schneeweiss A, Chia S, Hickish T et al (2013) Pertuzumab plus trastuzumab in combination with standard neoadjuvant anthracycline-containing and anthracycline-free chemotherapy regimens in patients with HER2-positive early breast cancer: a randomized phase II cardiac safety study (TRYPHAENA). Ann Oncol 24(9):2278-2284

25. Garrison LP, Babigumira J, Tournier C et al (2019) Cost-effectiveness analysis of pertuzumab with trastuzumab and chemotherapy compared to trastuzumab and chemotherapy in the adjuvant treatment of HER2-positive breast cancer in the United States. Value in Health 22(4):408-415

26. Paranjpe R, Basatneh D, Tao G et al (2019) Neratinib in HER2-positive breast cancer patients. Ann Pharmacother 53(6):612-620
27. Ejlertsen B, Barrios CH, Gokmen E et al (2018) Timing of initiation of neratinib after trastuzumab-based adjuvant therapy in early-stage HER2 hormone receptor (HR)-negative breast cancer: Exploratory analyses from the phase III ExteNET trial [Abstract]. J Clin Oncol 36(15 supp):549

28. Ustaris F, Saura C, Di Palma J et al (2015) Effective management and prevention of neratinib-induced diarrhea. Am J Hematol Oncol 11(11):13-22

29. Hurvitz S, Chan A, Iannotti N et al (2017) Effects of adding budesonide or colestipol to loperamide prophylaxis on neratinib-associated diarrhea in patients with HER2+ early-stage breast cancer: the CONTROL trial. San Antonio Breast Cancer Symposium, San Antonio, TX

30. Schwartz NR, Flanagan MR, Babigumira JB et al (2019) Costeffectiveness analysis of adjuvant neratinib following trastuzumab in early-stage HER2-positive breast cancer. J Manag Care Spec Pharm 25(10):1133-1139

Publisher's Note Springer Nature remains neutral with regard to jurisdictional claims in published maps and institutional affiliations. 